\title{
Plasmonic Analog of Electromagnetically Induced Absorption Leads to Giant Thin Film Faraday Rotation of $14^{\circ}$
}

\author{
Dominik Floess, ${ }^{1, *}$ Mario Hentschel, ${ }^{1}$ Thomas Weiss, ${ }^{1}$ Hanns-Ulrich Habermeier, ${ }^{2}$ \\ Jian Jiao, ${ }^{1}$ Sergei G. Tikhodeev, ${ }^{3}$ and Harald Giessen ${ }^{1}$ \\ ${ }^{1} 4$ th Physics Institute and Research Center SCoPE, University of Stuttgart, Stuttgart 70569, Germany \\ ${ }^{2}$ Max Planck Institute for Solid State Research, 70569 Stuttgart, Germany \\ ${ }^{3}$ Department of Physics, Lomonosov Moscow State University, Moscow 119991, Russia \\ and A. M. Prokhorov General Physics Institute, Russian Academy of Sciences, Moscow 119991, Russia
}

(Received 6 February 2017; revised manuscript received 17 April 2017; published 27 June 2017)

\begin{abstract}
We demonstrate the realization of a new hybrid magnetoplasmonic thin film structure that resembles the classical optical analog of electromagnetically induced absorption. In transmission geometry our gold nanostructure embedded in an EuS film induces giant Faraday rotation of over $14^{\circ}$ for a thickness of less than $200 \mathrm{~nm}$ and a magnetic field of $5 \mathrm{~T}$ at $T=20 \mathrm{~K}$. By varying the magnetic field from -5 to $+5 \mathrm{~T}$, a rotation tuning range of over $25^{\circ}$ is realized. As we are only a factor of 3 away from the Faraday isolation requirement, our concept could lead to highly integrated, nonreciprocal photonic devices for light modulation, optical isolation, and optical magnetic field sensing.
\end{abstract}

DOI: 10.1103/PhysRevX.7.021048

Subject Areas: Optics, Photonics, Plasmonics

\section{INTRODUCTION}

The combination of plasmonic nanostructures with magneto-optic (MO) materials enables nanoscale systems that offer magnetic tunability as well as strong nonreciprocal optical response in ultracompact structures [1-6]. In recent years, the demonstration of plasmonically enhanced $\mathrm{MO}$ effects, such as the MO Kerr effect [7-11] and the Faraday effect [12-14], have received considerable attention. Here, the Faraday effect is of special interest as it is the potentially largest MO effect. It describes the phenomenon that the polarization plane of linearly polarized light propagating through a material is rotated by an angle that is proportional to the applied static magnetic field and to the materialspecific Verdet constant $[15,16]$. A very intriguing and unique property of the Faraday effect is that time-reversal symmetry and Lorentz reciprocity are broken [17]. As a result, the direction of the polarization rotation is determined by the direction of the applied magnetic field and not by the direction of the wave vector of the incident light. This fact fundamentally distinguishes it from effects such as optical activity [18]. MO effects are actually the only practical way to break Lorentz reciprocity in passive optical systems, since other approaches rely on either nonlinear effects [19-21] or time modulation [22-26]. For that reason, Faraday rotators

\footnotetext{
*Corresponding author. d.floess@pi4.uni-stuttgart.de

Published by the American Physical Society under the terms of the Creative Commons Attribution 4.0 International license. Further distribution of this work must maintain attribution to the author(s) and the published article's title, journal citation, and DOI.
}

are widely utilized as core elements in nonreciprocal optical devices such as optical isolators [27]. As such, Faraday rotators are essential components in a multitude of optical systems, including optical telecommunication networks [28-30] and laser systems [31-33]. Furthermore, Faraday rotators are attractive for magnetic field sensing $[34,35]$ and optical modulation [36].

Most systems that involve Faraday rotators have recently undergone massive miniaturization. Hence, there is a big demand for down-sized Faraday rotators [37,38]. However, such systems are very challenging to realize since Faraday rotation is proportional to the thickness of the MO material. The first approaches to solve this problem were based on $\mathrm{MO}$ nanoparticles [12], photonic crystals [39-43], and microcavities [44-46] but were hampered by either still weak MO effects or relatively large structure sizes that are also difficult to fabricate. Considerable attention was received by a recent approach where the MO response of a dielectric thin film is enhanced by the attachment of a plasmonic grating $[13,14]$. This technique also allows the amplified MO response to be spectrally tailored by tuning the grating parameters [47]. Very recently, the underlying enhancement mechanism has also been described analytically using a simple oscillator model based on the Lorentz force [48]. However, the MO performance of such a system is limited by the low $Q$ factor of the plasmon resonances of the grating.

\section{PLASMONIC ANALOG OF ELECTROMAGNETICALLY INDUCED ABSORPTION (EIA)}

This limitation is lifted elegantly by our novel approach, which is based on a classical optical analog of electromagnetically induced absorption (EIA) [49,50]. Here, a 
strongly damped plasmon oscillation is weakly coupled to a narrow linewidth waveguide resonance with a phase delay, leading to constructive interference. By tuning this coupling carefully, a high- $Q$ absorptive hybrid mode is realized, which can be used to resonantly amplify the Faraday rotation response. Furthermore, the EIA mechanism allows us to utilize the high oscillator strength of the plasmonic resonance, leading to an efficient coupling of the incident light into the structure without reducing the effective $Q$ factor due to the broad plasmonic resonance, as was the case in previous approaches $[7,14,47]$. Although being only less than $200 \mathrm{~nm}$ thick, our novel structure design exhibits an order of magnitude better rotation capability than previous Ansätze that resulted in only fractions of degree rotation [13,14,41-43]. Furthermore, for low magnetic fields, our approach still yields rotation angles that were only achievable with 20 times stronger field strength in previous approaches [47]. The performance of our thin film structure is only a factor of 3 away from the $45^{\circ}$ that are required for building a Faraday isolator, which usually requires $\mathrm{MO}$ media with a thickness on the order of centimeters. The first demonstration of plasmonic resemblances of EIA [51,52] and its related effect, namely, electromagnetically induced transparency (EIT) [53-55], triggered significant attention. The special dispersion properties of such systems are known to facilitate plasmonic sensing with narrow linewidths [56-58] as well as slow light [59-61] and delay lines $[62,63]$. Furthermore, there have been extensive studies on enhanced nonlinear response $[64,65]$ in such systems. Here, we demonstrate that the EIA-like optical dispersion in our system also facilitates a giant $\mathrm{MO}$ response. While in EIT the light within a narrow spectral band passes through the sample, in the case of EIA real material polarization is excited within a narrow spectral region.

Another important aspect of our approach is the introduction of EuS as a new dielectric material for hybrid magnetoplasmonics. At low temperatures, EuS possesses an exceptionally large Verdet constant in combination with a high saturation magnetic flux density, resulting in a potentially very strong MO response [66,67]. Furthermore, it is transparent in the visible, qualifying it for utilization in Faraday geometry. While EuS possesses a similar refractive index and absorption coefficient to EuSe [66,67], EuS is significantly cheaper and already exhibits a stronger $\mathrm{MO}$ response at low magnetic field strengths. Further information about the temperature dependence of the EuS material parameters can be found in the Supplemental Material [68]. Despite the need for low temperatures, EuS is well suited for studying plasmonic MO model systems, as it combines the above-mentioned benefits with simple fabrication by physical vapor deposition. This allows for the realization of complex hybrid magnetoplasmonic structure geometries, which are challenging when fabricated with commonly used magneto-optic materials, such as bismuth iron garnet
[69] or yttrium iron garnet [70]. The reason is that the deposition of garnet films is a sophisticated process that usually involves pulsed laser deposition and hightemperature annealing that could damage any underlying plasmonic structures.

Our structure geometry is depicted in Figs. 1(a) and 1(b). It consists of an $\mathrm{EuS}$ thin film with an embedded $\mathrm{Au}$ nanowire grating. The EuS film is magnetized by an external magnetic field in the $z$ direction, which is also the direction of propagation of the incident light. The structure is fabricated in a three-step process. First, the bottom layer of the EuS film with thickness $b$ is evaporated onto the glass substrate by physical vapor deposition. After that, the gold wire grating with thickness $t$, width $w$, and period $p$ is structured via electron beam lithography. In the last step, an EuS film with thickness $(h-b)$ is evaporated. This results in an EuS-Au thin film structure with a slightly corrugated upper surface. Figure 1(c) shows a colored scanning electron micrograph of the cross section of such a structure with geometry parameters $p=490 \mathrm{~nm}$, $t=33 \mathrm{~nm}, w=85 \mathrm{~nm}, h=139 \mathrm{~nm}$, and $b=33 \mathrm{~nm}$. For the case of $b=0$, the non-MO dispersion properties of such a metal-dielectric hybrid structure have previously been analyzed in detail by Zentgraf et al. [71]. Because of the presence of the glass substrate with a higher refractive index than the air above the sample, the $x$ component of the electric field of the TM waveguide mode is concentrated in the upper part of the EuS waveguide. Hence, the coupling between plasmon and TM waveguide mode is weak in comparison to the case where the metal wires are attached on top. In the work by Zentgraf et al., it was also demonstrated that this weak coupling can lead to the
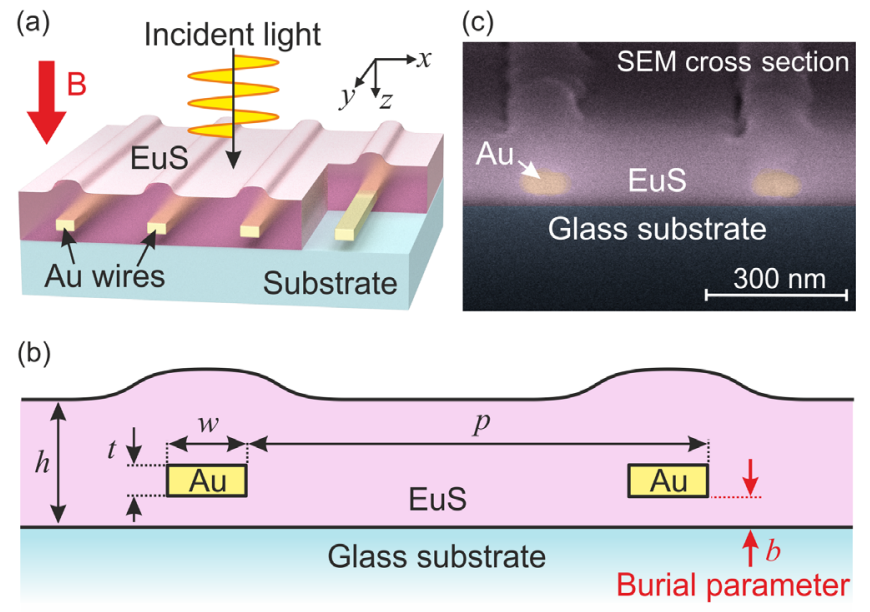

FIG. 1. (a),(b) Schematic drawing of the sample geometry. $p$, nanowire period; $t, w$, gold nanowire thickness and width. The wires are buried with a distance $b$ between the glass substrate and their lower edge. The nominal thickness of the EuS magnetooptical waveguide is $h$, which is increased near the position of the gold nanowires. (c) Colorized scanning electron micrograph of the sample cross section. The samples are measured at $T=20 \mathrm{~K}$. 
classical analog of the quantum mechanical effect of electromagnetically induced transparency, resulting in a narrow transmission peak on top of a broad transmission dip for $x$-polarized incident light.

We now demonstrate that by increasing the burial parameter $b$ in our hybrid system, the phase between the plasmonic mode and the TM waveguide mode can be tuned such that the system undergoes a transition from EIT-like to EIA-like behavior [51,52]. Figure 2 displays the simulated transmittance and absorbance spectra for $x$-polarized incident light and different burial parameters $b$. The simulations have been carried out using the Fourier modal method for anisotropic materials [72]. Our implementation is based on a scattering matrix algorithm [73], which has been improved by adaptive spatial resolution [74] to enable an efficient derivation of optical properties of metallodielectric systems. The far-field spectra are derived from the small scattering matrix, which provides the reflectance and transmittance for all diffracted orders, i.e., for all open far-field channels. The refractive index of the substrate is assumed to be 1.456, and for the gold wires the permittivity
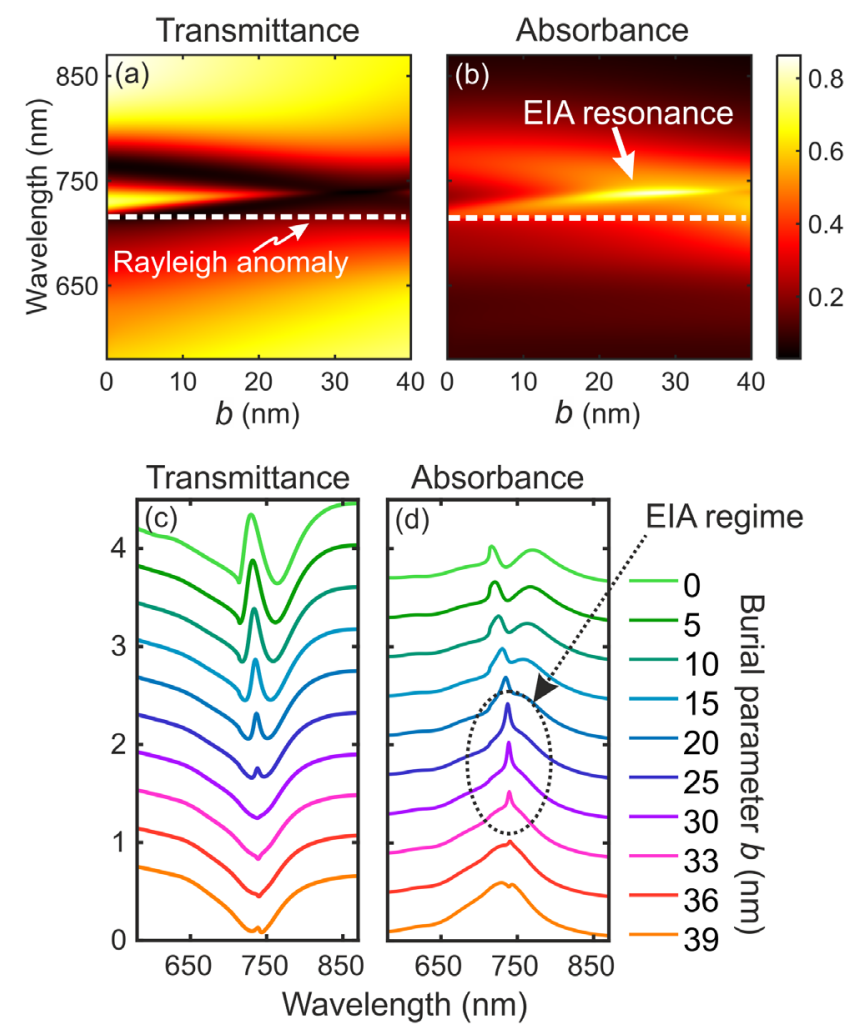

FIG. 2. (a),(b) Simulated transmittance and absorbance ( $1-T-R$, with $T$ and $R$ denoting transmittance and reflectance, respectively) for $x$-polarized incident light and different burial parameters $b$. For increasing $b$ there is a gradual transition from the regime of induced transparency to induced absorption. The white dotted line indicates the Rayleigh anomaly. (c),(d) Slice cuts for a clearer view on the line shapes of the spectra. The oval indicates the EIA regime, characterized by very sharp induced absorption peaks. data by Johnson and Christy [75] are used. In the investigated wavelength range, the refractive index of EuS is approximately 2 . The utilized material model parameters for EuS can be found in the Supplemental Material [76]. The geometry parameters are set to $p=490 \mathrm{~nm}$, $t=33 \mathrm{~nm}, w=85 \mathrm{~nm}$, and $h=139 \mathrm{~nm}$. In all simulations the bumps in the EuS film above the Au wires are approximated by a rectangular shape with thickness $t$ and a width of $2 w$. As expected [71], for small values of $b$, the transmittance spectrum exhibits the characteristic EIT line shape with a broad, mainly plasmon-induced dip and a narrow, mainly TM waveguide-induced peak. For increasing values of $b$, this effect is reversed and the sharp waveguide feature flips around, resulting in a sharp absorbance peak that is characteristic for an EIA-like system. At $715 \mathrm{~nm}$, the spectra are slightly distorted due to the Rayleigh anomaly [77]. The transition of the spectra with increasing $b$ can be understood in the picture of coupled Lorentz oscillators introduced by Taubert et al. [52]: Here, a strongly damped Lorentz oscillator (in this case the plasmon) and a less damped Lorentz oscillator (in this case the TM waveguide mode) are coupled via a complex coupling constant that includes an additional phase delay. It was shown that by changing the phase of the coupling constant, the optical response of the system turns from EIT-like to an EIA-like behavior. In our case, this behavior is particularly pronounced for $b$ between 25 and $36 \mathrm{~nm}$ [see Fig. 2(d), oval area]. Furthermore, by changing the distance between the wire grating and the substrate, we can change the coupling phase. As in other cases of plasmonic EIT and EIA, the reflection behavior of the system also changes with the coupling phase, which leads to the situation that the EIT coupling regime can be observed most pronounced in the transmittance spectrum, whereas the EIA case can be identified best in the absorbance spectrum [52]. In Sec. III, we elaborate more on interpreting the magnetoplasmonic system in the picture of coupled oscillators.

For a burial parameter of $b=33 \mathrm{~nm}$, the EIA coupling regime manifests itself also in the transmittance spectrum in the form of a small dip [see Fig. 2(c)]. For this case the relation between the EIA-like behavior and the MO response is investigated experimentally. Figures 3(a) and 3(b) display the measured transmittance and Faraday rotation spectra for $x$-polarized incident light and different grating periods $p$. The measurements are performed at $20 \mathrm{~K}$ and a magnetic field of $5 \mathrm{~T}$ in the $z$ direction. The Faraday rotation is measured with a rotating analyzer setup where the incident polarization state is prepared using a polarizer inside the cryostat [47]. For a period of $490 \mathrm{~nm}$, the sharp EIA feature is best centered with respect to the broad, mainly plasmonic, transmittance feature. For the same grating period, at the spectral position of the EIA resonance, the Faraday rotation exhibits a sharp maximum of about $8^{\circ}$, which is a substantial rotation enhancement 


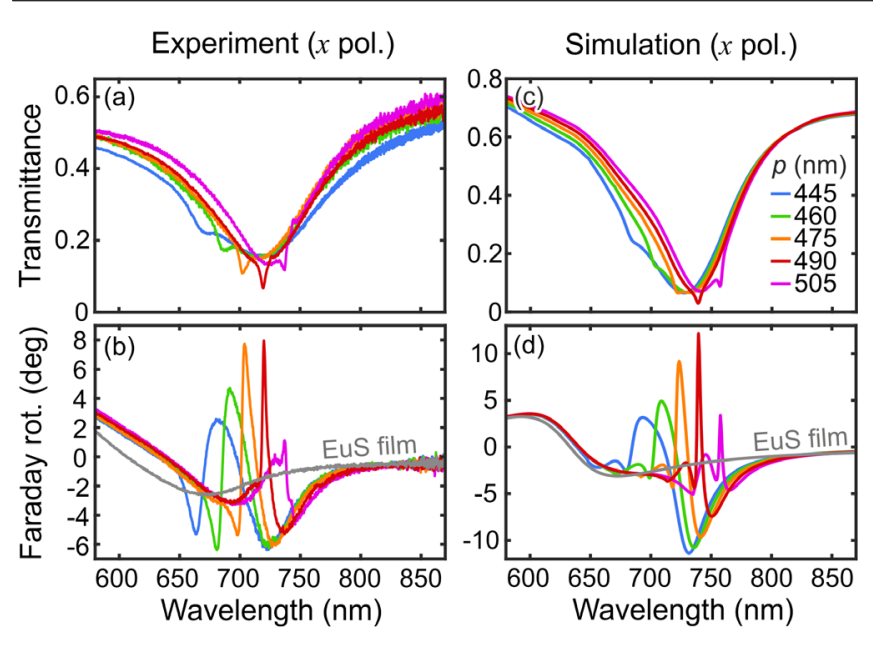

FIG. 3. The left-hand column displays the measured transmittance (a) and Faraday rotation (b) for $x$-polarized incident light for a wire width of $85 \mathrm{~nm}$, burial parameter $b=33 \mathrm{~nm}$, and different grating periods. The experimental data agree with the performed simulation shown in the right-hand column (c),(d).

compared to a bare EuS film with thickness $h=139 \mathrm{~nm}$ (gray curve). This behavior can be understood in the following way. For wavelengths close to the narrow EIA resonance, the system acts as a resonator with a relatively high $Q$ factor, implying multiple vertical round-trips of the light through the MO material before it is coupled out in the $z$ direction. Because of Lorentz nonreciprocity, the polarization rotation adds up for each propagation cycle, resulting in an enhanced rotation compared to a single pass through the EuS film. Furthermore, the EIA mechanism allows us to utilize the high oscillator strength of the plasmonic resonance, leading to an efficient coupling of the incident light into the structure without reducing the effective $Q$ factor due to the broad plasmonic resonance, as was the case in previous approaches $[7,14,47]$. As such, the plasmonic EIA mechanism provides an elegant way to greatly increase the interaction between the incident light and the MO material. In Sec. III, we discuss the connection between the EIA dispersion and Faraday rotation enhancement in further detail by utilizing a coupled oscillator model. The measurement is also in good agreement with the full numerical simulation displayed in Figs. 2(c) and 2(d). In the measured wavelength region, the Faraday rotation of EuS shows a notable wavelength dependence due to a material resonance (see gray line). Hence, in order to obtain realistic simulation results, we model the material dispersion of EuS with a MO Lorentz oscillator [78]. The free model parameters are fitted such that the measured MO response of a bare EuS film matches the simulated MO response in the wavelength range of interest between 650 and $850 \mathrm{~nm}$. More details on the EuS permittivity model can be found in the Supplemental Material [79]. The discrepancy between measured and simulated Faraday rotation below $650 \mathrm{~nm}$ is due to the limited model accuracy in this range. In the transmittance spectra, the grating-induced Rayleigh anomaly is barely visible. For example, for $p=490 \mathrm{~nm}$ it occurs at $715 \mathrm{~nm}$ and is most visible in the simulated Faraday rotation, where it manifests itself by a small kink. However, the Rayleigh anomaly has no major influence on the Faraday rotation enhancement, as it is well separated from the enhancement by the EIA resonance.

Also for $y$-polarized incident light, large Faraday rotation angles can be realized. This is illustrated by Figs. 4(a) and 4(b), which depict the corresponding transmittance and Faraday rotation spectra for a sample geometry with $t=33 \mathrm{~nm}, w=75 \mathrm{~nm}, h=139 \mathrm{~nm}, b=33 \mathrm{~nm}$, and different grating periods $p$. Since a direct excitation of the plasmon resonance and TM waveguide resonance is only possible for $x$-polarized light, they have no discernable influence on the transmittance line shape. However, as the $x$ - and $y$-polarized eigenmodes are coupled via the magnetic field, the Faraday rotation spectrum for $y$-polarized incidence is strongly influenced by the $x$-polarized eigenmodes (and vice versa) [48], which results in strongly enhanced Faraday rotation also for $y$-polarized incidence. Furthermore, this behavior is discussed in more detail in Sec. III. For a grating period of $505 \mathrm{~nm}$, the Faraday rotation reaches values of up to $14^{\circ}$ at a transmittance value of 17\%. Given the thin structure profile of below $200 \mathrm{~nm}$, this is a giant Faraday rotation value and only a factor of 3 away from a Faraday isolator. Again, the measurement results are in good agreement with the numerical simulations plotted in Figs. 4(c) and 4(d), except that the Rayleigh anomalies (kinks in the left shoulders of the transmittance spectra) are washed out in the measurement. This happens certainly due to sample roughness and limited fabrication accuracy. As in the case of $x$-polarized
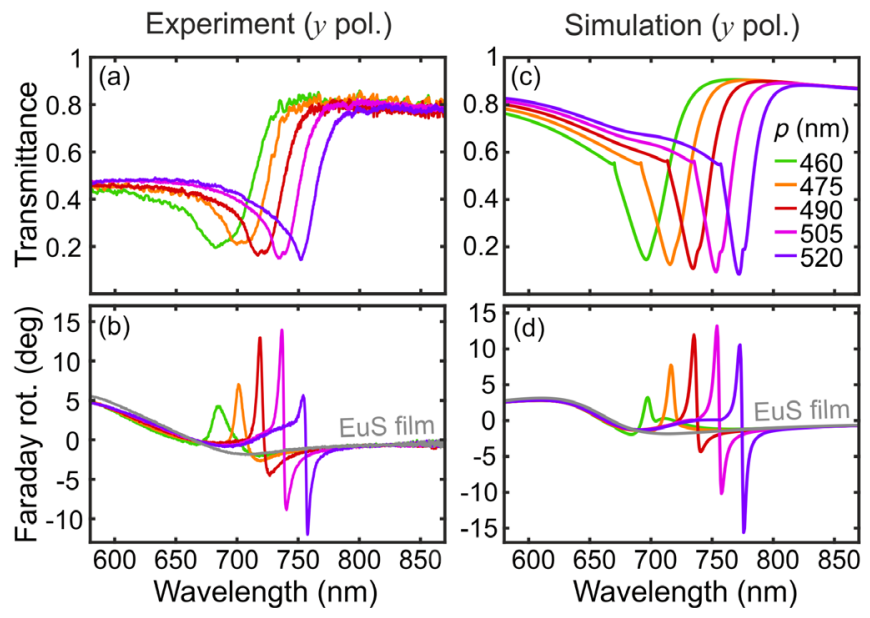

FIG. 4. The left-hand column displays the measured transmittance (a) and Faraday rotation (b) for $y$-polarized incident light for a wire width of $75 \mathrm{~nm}$, burial parameter $b=33 \mathrm{~nm}$, and different grating periods $p$. The experimental data agree well with the performed simulation shown in the right-hand column (c),(d). For a period of $505 \mathrm{~nm}$, the Faraday rotation reaches values of up to $14^{\circ}$. 

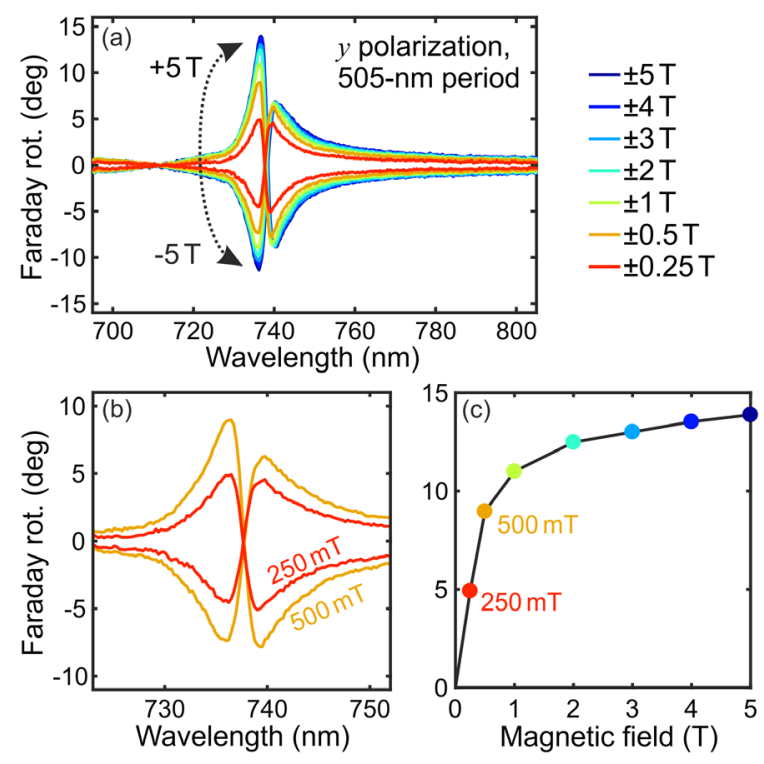

FIG. 5. (a) Measurement of the magnetic field dependence of the polarization rotation for a period of $505 \mathrm{~nm}$. (b) Closer view on the rotation spectra for weak magnetic fields. Already for $250 \mathrm{mT}$ the Faraday rotation reaches values of over $4^{\circ}$. (c) Saturation behavior of the Faraday rotation at $737 \mathrm{~nm}$.

incidence, the Rayleigh anomalies are well separated from the Faraday rotation enhancement feature and have no significant influence on the Faraday rotation spectra.

Furthermore, since the rotation angle scales with the applied magnetic field, our structure offers an impressive polarization tuning range of over $25^{\circ}$. This is indicated in Fig. 5(a), where the Faraday rotation spectra are plotted for different magnetic field strengths ranging from -5 to $+5 \mathrm{~T}$. The spectra for inverted magnetic fields exhibit almost perfectly mirror symmetric shapes. Any deviations from absolute mirror symmetry can be explained by a slight misalignment between the gold wires and the incident polarization. Figure 5(b) shows a close-up of the Faraday rotation enhancement feature for lower magnetic field strengths that can be realized easily using standard permanent magnets [80]. Even for magnetic fields as low as $250 \mathrm{mT}$, the Faraday rotation still reaches values of up to $4.9^{\circ}$. This is a similar rotation performance as was previously realized for a 220 -nm thick hybrid structure based on EuSe, however, only with 20 times larger magnetic fields [47]. Figure 5(c) displays the Faraday rotation at $737 \mathrm{~nm}$ as a function of applied magnetic field and illustrates the saturation behavior. Up to $500 \mathrm{mT}$ the rotation angle increases linearly with the applied magnetic field, whereas for larger fields saturation sets in and the response curve flattens out.

\section{HARMONIC OSCILLATOR MODEL}

To obtain further insight into the connection between EIA-like modal coupling and the enhancement of the magneto-optic response, it is useful to view our magnetoplasmonic system in the picture of coupled oscillators, as depicted in Fig. 6(a). This oscillator scheme was originally introduced in Ref. [48] and is now modified in analogy to the non-magneto-optic model by Taubert et al. to account for EIA [52]. In this very simple model, our system is described using two charged oscillators that are driven by the external light field: The oscillator on the right-hand side represents the plasmonic excitation, and its movement is restricted to the $x$ direction, as the plasmonic resonance can only be excited for $x$-polarized light. The second oscillator is associated with the waveguide excitations inside the MO slab. This oscillator can be displaced both in the $x$ and $y$ direction, which corresponds to the excitation of TM and TE polarized waveguide modes, respectively. As discussed by Taubert et al., it is crucial for realizing the coupling regime of EIA that the coupling between the two contributing oscillators has to include a retardation phase [51]. Hence, we model the coupling between plasmon and TM waveguide with a complex coupling constant $\tilde{\kappa}=\kappa \exp (i \phi)$, with $\phi$ as the phase. The influence of the applied static magnetic field is taken into account via the Lorentz force acting on the 2D waveguide oscillator.

(a)

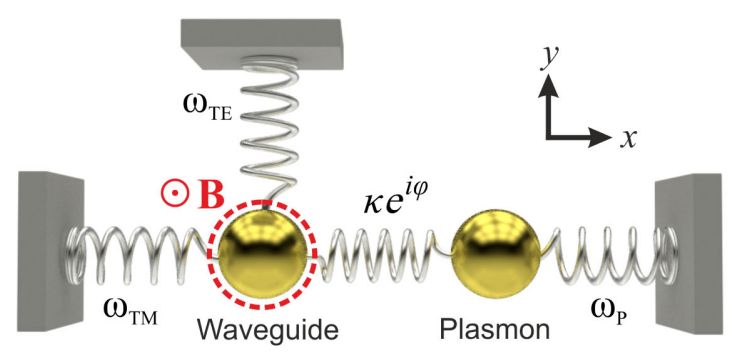

(b)
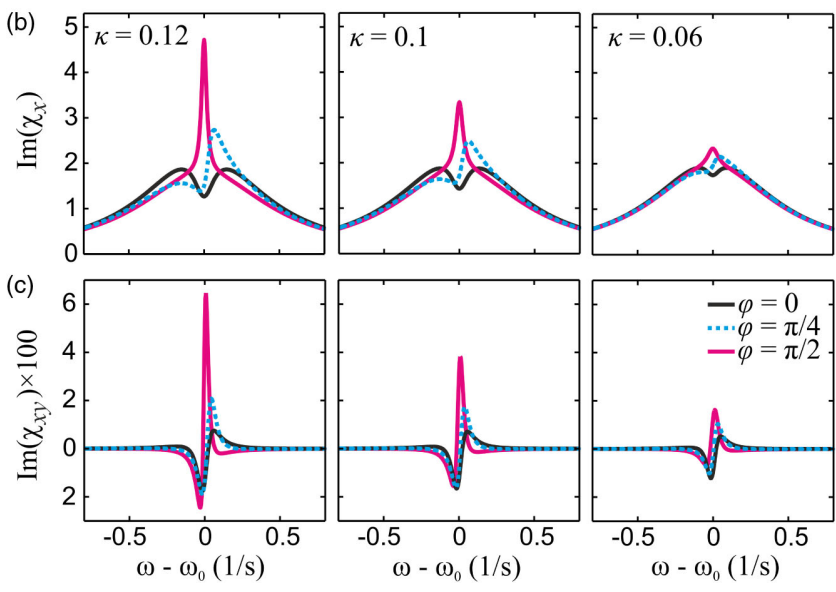

FIG. 6. (a) Oscillator model representing the magnetoplasmonic nanostructure. A precondition for EIA is that the coupling between the TM waveguide mode and the plasmon mode is weak and includes retardation. This is taken into account by the complex coupling constant $\kappa \exp (i \phi)$ with phase $\phi$. (b) Imaginary part of the component $\chi_{x}$, which is proportional to the absorbance of $x$-polarized incident light. (c) Imaginary part of $\chi_{x y}$, which induces polarization rotation of both $x$ - and $y$-polarized incident light. 
The resulting electric susceptibility associated with this oscillator system is given by [48]

$$
\chi(\omega)=\left(\begin{array}{cc}
\chi_{x} & +\chi_{x y} \\
-\chi_{x y} & \chi_{y}
\end{array}\right)=\eta R^{T} M^{-1} R,
$$

where we introduce the matrices

$$
M=\left(\begin{array}{ccc}
\Omega_{\mathrm{TM}} & -\kappa e^{i \phi} & -i \beta \\
-\kappa e^{i \phi} & \Omega_{\mathrm{P}} & 0 \\
+i \beta & 0 & \Omega_{\mathrm{TE}}
\end{array}\right)
$$

and

$$
R=\left(\begin{array}{cc}
\rho_{\mathrm{TM}} & 0 \\
\rho_{\mathrm{P}} & 0 \\
0 & \rho_{\mathrm{TE}}
\end{array}\right)
$$

Here, the terms $\Omega_{i}=\omega_{i}-\omega-i \gamma_{i} / 2(i=\mathrm{TM}, \mathrm{P}, \mathrm{TE})$ contain the eigenfrequencies and damping coefficients of the individual oscillators. The magneto-optic coefficient $\beta$ is proportional to the gyration of the waveguide material and, thus, to the applied magnetic field. The occurrence with two different signs originates from the cross product in the Lorentz force. The nonzero components $\rho_{i}(i=\mathrm{TM}, \mathrm{P}, \mathrm{TE})$ in the matrix $R$ are proportional to the charge of the oscillators and relate to the individual oscillator strengths. $\eta$ is a proportionality constant.

In the following, we use this model to illustrate the relation between the EIA and EIT coupling regimes as well as their influence on the magneto-optic response. For this, to keep the degrees of freedom to a minimum, the following simple (and dimensionless) parameter constellation is utilized: The individual resonances in our system are spectrally close, while the waveguide modes having significantly smaller linewidths than the plasmon. Hence, the eigenfrequencies of the three oscillators are assumed to be equal, i.e., $\omega_{\mathrm{TM}}=\omega_{\mathrm{TE}}=\omega_{\mathrm{P}}=\omega_{0}$, and for the damping coefficients we assume $\gamma_{\mathrm{P}}=1$ and $\gamma_{\mathrm{TM}}=\gamma_{\mathrm{TE}}=0.1$. Furthermore, we set $\eta=1$ and assume that the plasmon oscillator couples much stronger to the external light field than to the TM waveguide mode and set $\rho_{\mathrm{TM}}=0$ and $\rho_{\mathrm{P}}=1$. The oscillator strength of the TE waveguide oscillator is set to $\rho_{\mathrm{TE}}=0.32$, which represents the case in which $\chi_{x}$ and $\chi_{y}$ are approximately equal (see Supplemental Material [81]).

The plots in Fig. 6(b) show the resulting frequency dependence of the imaginary part of $\chi_{x}$, which is proportional to the absorbance of $x$-polarized incident light. The different line colors correspond to the different coupling phases. Furthermore, from the left to the right column, the coupling amplitude $\kappa$ is decreased. All spectra of $\chi_{x}$ show a broad, mainly plasmon-induced, background with a sharp modulation on top. For $\phi=0$, this modulation is a narrow dip at $\omega_{0}$ and corresponds to the case of EIT. Increasing the coupling phase to $\phi=\pi / 2$ turns this modulation into a narrow EIA resonance, while the case of $\phi=\pi / 4$ represents an intermediate regime. Furthermore, an increase of $\kappa$ leads to a more pronounced modulation. However, at some point when $\kappa$ is increased further (not shown here), the coupling is not weak anymore and a significant mode splitting occurs for $\phi=0$, and for $\phi=\pi / 2$ nonphysical solutions with negative absorption can emerge [52]. In summary, the classical analogs of EIT and EIA are indeed very closely related: Both scenarios occur in the regime of weak coupling where there is no significant spectral repulsion between the narrow linewidth mode (in this case, the TM waveguide mode) and the broad linewidth mode (in this case, the plasmon). The only difference between the two scenarios lies in the coupling phase $\phi$. We note at this point that the absorption behavior is approximately independent of the magnetic field, as $|\beta|$ can be assumed to be small (here, it is set to 0.001), in which case it predominantly influences the off-diagonal elements of $\chi$ but not the diagonal elements [48].

The corresponding magneto-optic response is encoded in the off-diagonal elements of $\chi$ that are proportional to $\beta$ and, thus, to the magnetic field. In first approximation, the Faraday rotation of the system is proportional to the imaginary part of $\chi_{x y}$ [82], which is plotted in Fig. 6(c). We note in passing that the real part of $\chi_{x y}$ would correspond to the ellipticity (not plotted here). The graphs nicely illustrate the fundamental working principle of our system: With increasing $\kappa$, not only the modulation of $\chi_{x}$ increases (i.e., the EIA or EIT resonances become more pronounced), but also the modulation of $\chi_{x y}$ becomes stronger. In other words, due to the magnetic field, the EIA or EIT resonances not only occur in absorption, but also in Faraday rotation. The more pronounced the EIA or EIT resonances are, the larger the Faraday rotation enhancement becomes. Furthermore, for a constant $\kappa$ this modulation of $\chi_{x y}$ is strongest for $\phi=\pi / 2$, i.e., for the EIA case. At this point we should note that in practice the difference in the MO response obtained by EIA-like and EIT-like coupling can be less pronounced than in the modeled spectra. The reason is that tuning the structure geometry from the EIT to the EIA case usually also slightly influences the other coupling parameters such as $\kappa$, which also have strong influence on $\chi_{x y}$. However, the model correctly reflects the trend that the EIA case produces stronger MO response than the EIT case. Another revealing aspect of this model is that, although only the oscillators moving in the $x$ direction contribute to the EIA resonance, this resonance also leads to increased polarization rotation for $y$-polarized light: This follows from the fact that $\chi$ is antisymmetric. Hence, the resonance in $\chi_{x y}$ translates to a conversion from $x$ to $y$ polarization as well as to a conversion from $y$ to $x$ polarization. However, in general, the Faraday rotation spectra for $x$ - and $y$-polarized light are 
not completely equal since for finite propagation distances also the diagonal components $\chi_{x}$ and $\chi_{y}$ possess an influence $[48,82]$. As shown by the measurements in Fig. 4, the Faraday rotation can be even larger for $y$-polarized light than for $x$-polarized light. We also point out that the line shape of $\chi_{x y}$ depends on the complex phase of the magneto-optical coupling constant $\beta$, which is related to the gyration of the MO material [48]. For the present example, this phase is set to $-45^{\circ}$. Changing this phase does not affect the average magnitude of the magneto-optic response, but only determines whether the $\operatorname{Im}\left(\chi_{x y}\right)$ spectra show a down-up line shape (negative phase), an up-down line shape (positive phase), or a peak (zero phase) when plotted over angular frequency [83].

As a final remark, we mention that the presented oscillator model is the simplest possible approach to illustrate the relation between EIA-like coupling and the enhanced MO response in our system. Of course, this results in neglecting secondary influences observed in the actual measurements, such as material dispersion, background Faraday rotation due to the film, or diffraction effects such as Rayleigh anomalies [48]. Nevertheless, the benefit of this model is that it provides a very intuitive description of our system.

\section{CONCLUSION}

We exploit the design flexibility enabled by the use of EuS as magneto-optic material to realize a hybrid magnetoplasmonic thin film structure that represents the classical optical analog of EIA. Unlike in previous approaches where the low quality factor of localized surface plasmon resonances limited the Faraday rotation enhancement, the coupling regime of EIA allows us to leverage both the highquality factor of the waveguide resonances and the large oscillator strength of the plasmons. Both these aspects result in dramatically increased light-matter interaction and MO response. In relation to its thickness, our system exhibits giant Faraday rotation response that substantially exceeds the results of previous approaches. Furthermore, compared to EuSe-based structures, our approach reaches similar performance already at much lower magnetic fields. A fruitful strategy to even further increase the MO response of our system could be to use a thicker MO slab in combination with grating periods that are comparable to the ones we use in this work. This way, higher-order waveguide resonances can be excited, which in combination with EIA-like dispersion could lead to Faraday rotation values closer to or even above $45^{\circ}$. Furthermore, some sort of physical or chemical treatment to enhance the surface quality of the EuS film could also further increase the resonator performance resulting in larger Faraday rotation. In future designs, the necessity for low temperatures could be significantly relaxed by doping europium compounds with gadolinium. For example, it has been shown that alloying $\mathrm{EuO}$ with gadolinium can raise the Curie temperature (where the Faraday rotation starts to drop) from 69 to $135 \mathrm{~K}[84,85]$, which is well above liquid nitrogen temperature. Of course, the concept of an EIAboosted $\mathrm{MO}$ response is not restricted to $\mathrm{EuS}$ or other $\mathrm{Eu}$ compounds and can, in principle, be applied to many materials. The only requirements are sufficient transparency of the MO material and a suitable procedure for the nanostructure fabrication. Furthermore, as was demonstrated recently, strong MO dispersion of nanostructured systems can also be leveraged for refractive index sensing, allowing very precise detection of biochemical substances [86]. Here, the exceptionally sharp EIA-induced features in the MO spectra of our system could also turn out to be a powerful tool. With all that, we believe that our concept can lead to highly integrated, nonreciprocal nanophotonic devices for light modulation, optical isolation, and optical magnetic field sensing. Moreover, we prove that EuS enables simple fabrication of complex layer-based magneto-optical geometries. This is a very powerful and rare property among transparent magneto-optic materials. Since EuS is much cheaper than EuSe and also provides stronger MO response already at low magnetic fields, EuS could become a trigger for other interesting and potentially very powerful magneto-optic and magnetoplasmonic designs. This could include both 2D and 3D systems, for instance, EuS photonic crystals, as well as even magneto-optic metamaterials $[38,87]$.

\section{ACKNOWLEDGMENTS}

The authors acknowledge support from DFG (SPP 1839, Mercator-Professorship), BMBF (13N12443 Faraday), MWK Baden-Württemberg (Zukunftsoffensive IV), BW Stiftung (Spitzenforschung II, China exchange scholarship), ERC Complexplas, and RFBR (16-29-03283). Furthermore, the authors thank Manfred Bayer (TU Dortmund) for providing the cryostat system, Yvonne Link (MPI FKF Stuttgart) for the evaporation service, Audrey Berrier (Uni Stuttgart) for the ellipsometry, and Liwei Fu (Uni Stuttgart) for the FIB cutting. This publication was supported by the Open Access Publishing Fund of the University of Stuttgart.

[1] V. Temnov, G. Armelles, U. Woggon, D. Guzatov, A. Cebollada, A. Garcia-Martin, T. Thomay, A. Leitenstorfer, and R. Bratschitsch, Active Magneto-Plasmonics in Hybrid Metal-Ferromagnet Structures, Nat. Photonics 4, 107 (2010).

[2] V. I. Belotelov, I. A. Akimov, M. Pohl, V. A. Kotov, S. Kasture, A. S. Vengurlekar, A. V. Gopal, D. R. Yakovlev, A. K. Zvezdin, and M. Bayer, Enhanced Magneto-Optical Effects in Magnetoplasmonic Crystals, Nat. Nanotechnol. 6, 370 (2011).

[3] V. Belotelov, L. Kreilkamp, I. Akimov, A. Kalish, D. Bykov, S. Kasture, V. Yallapragada, A. Venu Gopal, A. Grishin, 
S. Khartsev, M. Nur-E-Alam, M. Vasiliev, L. Doskolovich, D. Yakovlev, K. Alameh, A. Zvezdin, and M. Bayer, Plasmon-Mediated Magneto-Optical Transparency, Nat. Commun. 4, 2128 (2013).

[4] A. R. Davoyan and N. Engheta, Nonreciprocal Rotating Power Flow within Plasmonic Nanostructures, Phys. Rev. Lett. 111, 047401 (2013).

[5] S. Fan, Nanophotonics: Magnet-Controlled Plasmons, Nat. Photonics 4, 76 (2010).

[6] A. B. Khanikaev, S. H. Mousavi, G. Shvets, and Y.S. Kivshar, One-Way Extraordinary Optical Transmission and Nonreciprocal Spoof Plasmons, Phys. Rev. Lett. 105, 126804 (2010).

[7] L. E. Kreilkamp, V. I. Belotelov, J. Y. Chin, S. Neutzner, D. Dregely, T. Wehlus, I. A. Akimov, M. Bayer, B. Stritzker, and Harald Giessen, Waveguide-Plasmon Polaritons Enhance Transverse Magneto-Optical Kerr Effect, Phys. Rev. X 3, 041019 (2013).

[8] B. Sepúlveda, J. B. González-Díaz, A. García-Martín, L. M. Lechuga, and G. Armelles, Plasmon-Induced MagnetoOptical Activity in Nanosized Gold Disks, Phys. Rev. Lett. 104, 147401 (2010).

[9] J. B. González-Díaz, B. Sepúlveda, A. García-Martín, and G. Armelles, Cobalt Dependence of the Magneto-Optical Response in Magnetoplasmonic Nanodisks, Appl. Phys. Lett. 97, 043114 (2010).

[10] J. C. Banthí, D. Meneses-Rodríguez, F. García, M. U. González, A. García-Martín, A. Cebollada, and G. Armelles, High Magneto-Optical Activity and Low Optical Losses in Metal-Dielectric Au/Co/Au-SiO(2) Magnetoplasmonic Nanodisks, Adv. Mater. 24, OP36 (2012).

[11] M. Kataja, T. K. Hakala, A. Julku, M. J. Huttunen, S. van Dijken, and P. Törmä, Surface Lattice Resonances and Magneto-Optical Response in Magnetic Nanoparticle Arrays, Nat. Commun. 6, 7072 (2015).

[12] P. K. Jain, Y. Xiao, R. Walsworth, and A. E. Cohen, Surface Plasmon Resonance Enhanced Magneto-Optics (SUPREMO): Faraday Rotation Enhancement in Gold-Coated Iron Oxide Nanocrystals, Nano Lett. 9, 1644 (2009).

[13] V. I. Belotelov, L. L. Doskolovich, and A. K. Zvezdin, Extraordinary Magneto-Optical Effects and Transmission through Metal-Dielectric Plasmonic Systems, Phys. Rev. Lett. 98, 077401 (2007).

[14] J. Y. Chin, T. Steinle, T. Wehlus, D. Dregely, T. Weiss, V. I. Belotelov, B. Stritzker, and H. Giessen, Nonreciprocal Plasmonics Enables Giant Enhancement of Thin-Film Faraday Rotation, Nat. Commun. 4, 1599 (2013).

[15] A. Buckingham and P. Stephens, Magnetic Optical Activity, Annu. Rev. Phys. Chem. 17, 399 (1966).

[16] P. R. Berman, Optical Faraday Rotation, Am. J. Phys. 78, 270 (2010).

[17] A. T. de Hoop, A Reciprocity Theorem for the Electromagnetic Field Scattered by an Obstacle, Appl. Sci. Res., Sect. B 8, 135 (1960).

[18] I. Tinoco, Advances in Chemical Physics (John Wiley \& Sons, Inc., New York, 2007), pp. 113-160.

[19] M. Soljaic, C. Y. Luo, J. D. Joannopoulos, and S. H. Fan, Nonlinear Photonic Microdevices for Optical Integration, Opt. Lett. 28, 637 (2003).
[20] S. Lepri and G. Casati, Asymmetric Wave Propagation in Nonlinear Systems, Phys. Rev. Lett. 106, 164101 (2011).

[21] I. V. Shadrivov, V. A. Fedotov, D. A. Powell, Y. S. Kivshar, and N. I. Zheludev, Electromagnetic Wave Analogue of an Electronic Diode, New J. Phys. 13, 033025 (2011).

[22] Z. Yu and S. Fan, Complete Optical Isolation Created by Indirect Interband Photonic Transitions, Nat. Photonics 3, 91 (2009).

[23] M. S. Kang, A. Butsch, and P. St. J. Russell, Reconfigurable Light-Driven Opto-Acoustic Isolators in Photonic Crystal Fibre, Nat. Photonics 5, 549 (2011).

[24] S. Fan, R. Baets, Al. Petrov, Z. Yu, J. D. Joannopoulos, W. Freude, A. Melloni, M. Popović, M. Vanwolleghem, D. Jalas, M. Eich, M. Krause, H. Renner, E. Brinkmeyer, and C. R. Doerr, Comment on "Nonreciprocal Light Propagation in a Silicon Photonic Circuit”, Science 335, 38 (2012).

[25] D. W. Wang, H. T. Zhou, M. J. Guo, J. X. Zhang, J. Evers, and S. Y. Zhu, Optical Diode Made from a Moving Photonic Crystal, Phys. Rev. Lett. 110, 093901 (2013).

[26] D. Sounas, C. Caloz, and A. Alù, Giant Non-Reciprocity at the Subwavelength Scale Using Angular Momentum-Biased Metamaterials, Nat. Commun. 4, 2407 (2013).

[27] C. Firby, P. Chang, A. Helmy, and A. Elezzabi, Magnetoplasmonic Faraday Rotators: Enabling Gigahertz Active Polarization Control for Integrated Plasmonics, ACS Photonics 3, 2344 (2016).

[28] M. Shirasaki and K. Asama, Compact Optical Isolator for Fibers Using Birefringent Wedges, Appl. Opt. 21, 4296 (1982).

[29] L. Bi, J. Hu, P. Jiang, and D. Kim, On-Chip Optical Isolation in Monolithically Integrated Non-Reciprocal Optical Resonators, Nat. Photonics 5, 758 (2011).

[30] L. Fan, J. Wang, L. T. Varghese, H. Shen, B. Niu, Y. Xuan, A. M. Weiner, and M. Qi, An All-Silicon Passive Optical Diode, Science 335, 447 (2012).

[31] I. Ikushima and M. Maeda, Self-Coupled Phenomena of Semiconductor Lasers Caused by an Optical Fiber, IEEE J. Quantum Electron. 14, 331 (1978).

[32] T. Morikawa, Y. Mitsuhashi, J. Shimada, and Y. Kojima, Return-Beam-Induced Oscillations in Self-Coupled Semiconductor Lasers, Electron Lett. 12, 435 (1976).

[33] K. Petermann, External Optical Feedback Phenomena in Semiconductor Lasers, IEEE J. Sel. Top. Quantum Electron. 1, 480 (1995).

[34] L. Sun, S. Jiang, and J. R. Marciante, All-Fiber Optical Magnetic-Field Sensor Based on Faraday Rotation in Highly Terbium-Doped Fiber, Opt. Express 18, 5407 (2010).

[35] M. N. Deeter, A. H. Rose, and G. W. Day, Fast, Sensitive Magnetic-Field Sensors Based on the Faraday Effect in YIG, J. Lightwave Technol. 8, 1838 (1990).

[36] W. E. Ross, Two-Dimensional Magneto-Optic Spatial Light Modulator for Signal Processing, Opt. Eng. (Bellingham, Wash.) 22, 485 (1983).

[37] M. Liu and X. Zhang, Plasmon-Boosted Magneto-Optics, Nat. Photonics 7, 429 (2013).

[38] A. F. Koenderink, A. Alù, and A. Polman, Nanophotonics: Shrinking Light-Based Technology, Science 348, 516 (2015). 
[39] M. J. Steel, M. Levy, and R. M. Osgood, High Transmission Enhanced Faraday Rotation in One-Dimensional Photonic Crystals with Defects, IEEE Photonics Technol. Lett. 12, 1171 (2000).

[40] A. A. Fedyanin, O. A. Aktsipetrov, D. Kobayashi, K. Nishimura, H. Uchida, and M. Inoue, Enhanced Faraday and Nonlinear Magneto-Optical Kerr Effects in Magnetophotonic Crystals, J. Magn. Magn. Mater. 282, 256 (2004).

[41] M. Diwekar, V. Kamaev, J. Shi, and Z. V. Vardeny, Optical and Magneto-Optical Studies of Two-Dimensional Metallodielectric Photonic Crystals on Cobalt Films, Appl. Phys. Lett. 84, 3112 (2004).

[42] B. Caballero, A. García-Martín, and J. C. Cuevas, Faraday Effect in Hybrid Magneto-Plasmonic Photonic Crystals, Opt. Express 23, 22238 (2015).

[43] B. Caballero, A. Garciá-Martín, and J. C. Cuevas, Hybrid Magnetoplasmonic Crystals Boost the Performance of Nanohole Arrays as Plasmonic Sensors, ACS Photonics 3, 203 (2016).

[44] R. Rosenberg, C. B. Rubinstein, and D. R. Herriott, Resonant Optical Faraday Rotator, Appl. Opt. 3, 1079 (1964).

[45] M. Inoue, K. Arai, T. Fujii, and M. Abe, One-Dimensional Magnetophotonic Crystals, J. Appl. Phys. 85, 5768 (1999).

[46] S. I. Khartsev and A. M. Grishin, High Performance $\left[\mathrm{Bi}_{3} \mathrm{Fe}_{5} \mathrm{O}_{12} / \mathrm{Sm}_{3} \mathrm{Ga}_{5} \mathrm{O}_{12}\right]^{m} \quad$ Magneto-Optical Photonic Crystals, J. Appl. Phys. 101, 053906 (2007).

[47] D. Floess, J. Y. Chin, A. Kawatani, D. Dregely, H.-U. Habermeier, T. Weiss, and H. Giessen, Tunable and Switchable Polarization Rotation with Non-Reciprocal Plasmonic Thin Films at Designated Wavelengths, Light Sci. Appl. 4, e284 (2015).

[48] D. Floess, T. Weiss, S. G. Tikhodeev, and H. Giessen, Lorentz Nonreciprocal Model for Hybrid Magnetoplasmonics, Phys. Rev. Lett. 117, 063901 (2016).

[49] A. M. Akulshin, S. Barreiro, and A. Lezama, Electromagnetically Induced Absorption and Transparency due to Resonant Two-Field Excitation of Quasidegenerate Levels in Rb Vapor, Phys. Rev. A 57, 2996 (1998).

[50] A. Lezama, S. Barreiro, and A. M. Akulshin, Electromagnetically Induced Absorption, Phys. Rev. A 59, 4732 (1999).

[51] R. Taubert, M. Hentschel, J. Kästel, and H. Giessen, Classical Analogue of Electromagnetically Induced Absorption in Plasmonics, Nano Lett. 12, 1367 (2012).

[52] R. Taubert, M. Hentschel, and H. Giessen, Plasmonic Analog of Electromagnetically Induced Absorption: Simulations, Experiments, and Coupled Oscillator Analysis, J. Opt. Soc. Am. B 30, 3123 (2013).

[53] S. E. Harris, Electromagnetically Induced Transparency, Phys. Today 50, No. 7, 36 (1997).

[54] K. J. Boller, A. Imamoglu, and S. E. Harris, Observation of Electromagnetically Induced Transparency, Phys. Rev. Lett. 66, 2593 (1991).

[55] N. Liu, L. Langguth, T. Weiss, J. Kästel, M. Fleischhauer, T. Pfau, and H. Giessen, Plasmonic Analogue of Electromagnetically Induced Transparency at the Drude Damping Limit, Nat. Mater. 8, 758 (2009).

[56] N. Liu, T. Weiss, M. Mesch, L. Langguth, U. Eigenthaler, M. Hirscher, C. Sönnichsen, and H. Giessen, Planar Metamaterial Analogue of Electromagnetically Induced
Transparency for Plasmonic Sensing, Nano Lett. 10, 1103 (2010).

[57] N. Verellen, P. Van Dorpe, C. Huang, K. Lodewijks, G. A. E. Vandenbosch, L. Lagae, and V. V. Moshchalkov, Plasmon Line Shaping Using Nanocrosses for High Sensitivity Localized Surface Plasmon Resonance Sensing, Nano Lett. 11, 391 (2011).

[58] J. B. Lassiter, H. Sobhani, J. A. Fan, J. Kundu, F. Capasso, P. Nordlander, and N. J. Halas, Fano Resonances in Plasmonic Nanoclusters: Geometrical and Chemical Tunability, Nano Lett. 10, 3184 (2010).

[59] M. F. Yanik, W. Suh, Z. Wang, and S. Fan, Stopping Light in a Waveguide with an All-Optical Analog of Electromagnetically Induced Transparency, Phys. Rev. Lett. 93, 233903 (2004).

[60] K. Totsuka, N. Kobayashi, and M. Tomita, Slow Light in Coupled-Resonator-Induced Transparency, Phys. Rev. Lett. 98, 213904 (2007).

[61] A. Schönhardt, D. Nau, C. Bauer, A. Christ, H. Gräbeldinger, and H. Giessen, Phase-Resolved Pulse Propagation through Metallic Photonic Crystal Slabs: Plasmonic Slow Light, Phil. Trans. R. Soc. A 375, 20160065 (2017).

[62] L. Maleki, A. B. Matsko, A. A. Savchenkov, and V. S. Ilchenko, Tunable Delay Line with Interacting WhisperingGallery-Mode Resonators, Opt. Lett. 29, 626 (2004).

[63] N. Papasimakis, V. A. Fedotov, N. I. Zheludev, and S. L. Prosvirnin, Metamaterial Analog of Electromagnetically Induced Transparency, Phys. Rev. Lett. 101, 253903 (2008).

[64] M. Fleischhauer and J. P. Marangos, Electromagnetically Induced Transparency: Optics in Coherent Media, Rev. Mod. Phys. 77, 633 (2005).

[65] B. Metzger, T. Schumacher, M. Hentschel, M. Lippitz, and H. Giessen, Third Harmonic Mechanism in Complex Plasmonic Fano Structures, ACS Photonics 1, 471 (2014).

[66] K. A. Gschneidner and L. Eyring, Handbook on the Physics and Chemistry of Rare Earths (North-Holland Publishing Company, Amsterdam, 1979), Vol. 2, pp. 507-574.

[67] G. Güntherodt, J. Schoenes, and P. Wachter, Optical Constants of the Eu Chalcogenides Above and Below the Magnetic Ordering Temperatures, J. Appl. Phys. 41, 1083 (1970).

[68] See Supplemental Material at http://link.aps.org/ supplemental/10.1103/PhysRevX.7.021048 for further details.

[69] T. Wehlus, T. Körner, S. Leitenmeier, A. Heinrich, and B. Stritzker, Magneto-Optical Garnets for Integrated Optoelectronic Devices, Phys. Status Solidi 208, 252 (2011).

[70] H. Hayashi, S. Iwasa, N. J. Vasa, and T. Yoshitake, Fabrication of Bi-Doped YIG Optical Thin for Electric Current Sensor by Pulsed Laser Deposition, Appl. Surf. Sci. 197-198, 463 (2002).

[71] T. Zentgraf, S. Zhang, R. F. Oulton, and X. Zhang, Ultranarrow Coupling-Induced Transparency Bands in Hybrid Plasmonic Systems, Phys. Rev. B 80, 195415 (2009).

[72] L. Li, Fourier Modal Method for Crossed Anisotropic Gratings with Arbitrary Permittivity and Permeability Tensors, J. Opt. A 5, 345 (2003).

[73] S. G. Tikhodeev, A. L. Yablonskii, E. A. Muljarov, N. A. Gippius, and T. Ishihara, Quasiguided Modes and Optical 
Properties of Photonic Crystal Slabs, Phys. Rev. B 66, 045102 (2002).

[74] T. Weiss, G. Granet, N. A. Gippius, S. G. Tikhodeev, and H. Giessen, Matched Coordinates and Adaptive Spatial Resolution in the Fourier Modal Method, Opt. Express 17, 8051 (2009).

[75] P. B. Johnson and R. W. Christy, Optical Constants of the Noble Metals, Phys. Rev. B 6, 4370 (1972).

[76] See Supplemental Material at http://link.aps.org/ supplemental/10.1103/PhysRevX.7.021048 for further details.

[77] A. Christ, T. Zentgraf, J. Kuhl, S. G. Tikhodeev, N. A. Gippius, and H. Giessen, Optical Properties of Planar Metallic Photonic Crystal Structures: Experiment and Theory, Phys. Rev. B 70, 125113 (2004).

[78] G. R. Fowles, Introduction to Modern Optics, 2nd ed. (Dover Publications, New York, 1975).

[79] See Supplemental Material at http://link.aps.org/ supplemental/10.1103/PhysRevX.7.021048 for further details.

[80] M. Sagawa, S. Fujimura, N. Togawa, H. Yamamoto, and Y. Matsuura, New Material for Permanent Magnets on a Base of Nd and Fe, J. Appl. Phys. 55, 2083 (1984).
[81] See Supplemental Material at http://link.aps.org/ supplemental/10.1103/PhysRevX.7.021048 for further details.

[82] V. A. Kotov, Modern Magnetooptics and Magnetooptical Materials (IOP Publishing Ltd, London, 1997).

[83] Because of the $\omega$-based mathematical structure of the model, the response curves were plotted over angular frequency. This should be kept in mind when the modeled spectra are compared with the measured spectra, which are plotted over wavelength.

[84] J. Schoenes and P. Wachter, Exchange Optics in Gd-Doped EuO, Phys. Rev. B 9, 3097 (1974).

[85] M. W. Shafer, Studies of Curie-Point Increases in EuO, J. Appl. Phys. 39, 588 (1968).

[86] N. Maccaferri, K. E. Gregorczyk, T. de Oliveira, M. Kataja, S. van Dijken, Z. Pirzadeh, A. Dmitriev, J. Åkerman, M. Knez, and P. Vavassori, Ultrasensitive and Label-Free MolecularLevel Detection Enabled by Light Phase Control in Magnetoplasmonic Nanoantennas, Nat. Commun. 6, 6150 (2015).

[87] G. Castaldi, V. Galdi, A. Alù, and N. Engheta, Nonlocal Transformation Optics, Phys. Rev. Lett. 108, 063902 (2012). 